359 Il ammelsterg, ïber den Berthierit ven Bräunsdorf.

\title{
Ueber den Berthierit von Bräunsdorf bei Freiberg;
}

\section{von $R$ a m melsberg in Berlin.}

Vor melureren dahrea entdechle Berthier in der Auvergne ein dem fewöbulichen Gruuspiessglanzerz sehr ähnliches Fossil, von welehem in diesen Annalen Bd. XIV, S. MJ lienntniss mitgetheilt ist. Es liess sick sciner Zusammenselzung nach als cine Verbindong ven 5 Al. Schwefeleiseu urit 2 Itomen Schwefelantionan (JFe $s+2 S b_{3} s_{s}$ ) hetrachten und crhicht von II a $\mathrm{i}-$ dinger den Namen Berthierit. Auch von den 9 anderen, später von Herthier eutfechen, diesen nahe stebenden Fossilien, isi am ohen angefiulirten Orte dic Rede gewesen.

Nun ist in der Crube , Neue llofinumf Gottes" zu Dräunstorf bei Freilory ron Breithaupt cin Fossil entuckt worden, welehes mit dem Bertbierit sebr übercinzustimmen schien. Das deutsche Fossil enthält ansser einer bleinen Menge Zinli ate.h Muas Mangan, welehes sich vor dem Lölhrohr entolechen lässil.

Der Gaug der Analyse erfalb sich aus der vermulheteu Zusammensetzung mit Bujehtighicit. Das zepulverte Mineral warde in Kïnigswasser anfgelöst, whei der eingeuengle Quarz zurüchlileb. Wer ausfesonderte Sehwefel wurle von der Flüssigkeit getrennt; diese nit Chlorbaryum zur Abseheiding dex pebilueten Sebwefelsänre versetat, und, mach Entfernung des Barytüborschusses nitlest Schwefelsäure, das Antimon durch cinen Strom ron Schwofelwasserstuligus nicdergeschlngen. Der Schwefelgehalt des getrockneten und gewogenen Niederschlags 
Anmmrlsberg, Bber den Berthierit van Bräunsdorf. $\mathbf{3 3 3}$ wurde, lureb Oxylation desselben mit Käuigswasser u. s. w. ermitlelt, nal die Quantität des Antimons dadus -h indirect gefunden. Eisen, Zinli und Mangan wurden aus der vam Schwefelantimon abfiltrirten Flüssigkeit. dureh Amuoniucusulfhydrat gefallt, in fönigswasser aufgelöst, und aus der mit Ammoniak genan gesättiglen Auflösung das Eisen durch bernsteinsaures Anmoniak entfernt, worauf Zink und Mangan wiederum in Schwefelmetalle, hierauf in seluwefelsaure Salze verwanlelt und durch kaustisches liali (anmäberul) getrenut wurden.

I.

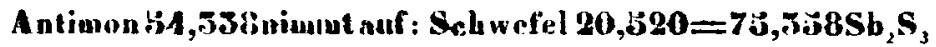
Eisen . 11,063 - . $\quad 7,006=10,061$ Fes Mangian 0,1665 • $\bullet \quad \bullet \quad 0,26:=0,721 \mathrm{MnS}$ Zink Spuren $97,8 8 1 \longdiv { 9 4 , 1 4 0 . }$

Schwefel no, 1573

07,754 .

II.

Antimon :\$A,700 nimmt aul: Schwefel20, $167=75,1685 \mathrm{~b}_{2} \mathrm{~s}$ Eisen . I1,170 . " . 6,780=18,212 Fes Mangan 2,544,$\quad$ 1,174=4,025 MnS Zink. 0,757
Schwefel 31.396 $\quad \frac{0,567=1,104 \mathrm{ZuS}}{29.065}$ 100,780.

In eiwem dritten Versuch ergahen sich 19,518 pCt. Eisen.

Betrachtet man Zink und Mangan als das Fisen zum Theil erselzend, so sicht man, dass die Sebwefelmengen, welche diese Mctalle zur Bildung der angegebenen Schwefelungsslufen bedürfen, sich en der im Schwefelantimon befindlichen fast wie 1 : 5 rerhalten, und es geht daraus die Formel 
334 Nammeloberg, Gber den Berthierit von Bräunsdorf.

$$
\left.\begin{array}{l}
\text { FeS } \\
\operatorname{MnS} \\
Z n S
\end{array}\right\} S_{b_{2}} S_{3}
$$

hervor. Bereehnet man dieselbe so, dass Zink und Mangan wegfallen (Fe Sh), so erhält man in 100 Theilen:

$$
\begin{aligned}
& \text { Antimon } . \quad 58,507=9 \text { At. } \\
& \text { Eisen . . . 12,505 }=1 \text {. } \\
& \text { Schwefel } \frac{99,148}{100}=1
\end{aligned}
$$

Der Berthierit von Bräunselorf ist folglich init dem von Auglar identisch.

\section{Neues Antimonerz aus Lappland.}

Thanlow aus Christiania hat ein neues Antimonerz untersueht, welches anfangs für Jninesouit gehalten wurdesich aber bei genauerer Untersuchung dennoch daron unterschied. Vor dem Löthrohr qualificirte so sich als eine Verbiulong von Schwefelantimon mit Schuefelblet. In diesen Sinne wurde die Aalyse mit trorkenem Chlorgase, nacb bekannten Verfahrungsarten, vorgenommen. Ohgleich dic Verbindung nicht gepulvert angewendet wurde, so war die Einwirkung dennoch so intensir, dass dic ganze Zersetzung obne Anwendung äussercr Wärme vor sich ging. Erst nach gesebehener Zersetzung wurden die Güchtifen Choride durch eine Weingeistlanne weffetrieben. Das Chorblei wurde in Wasser gelöst, wobei eine geringe Menge Kieselsäure zurückblieh, dann mit 